PROCEEDINGS OF THE

AMERICAN MATHEMATICAL SOCIETY

Volume 125, Number 9, September 1997, Pages 2767-2769

S 0002-9939(97)03893-8

\title{
NOTE ON FAITHFUL REPRESENTATIONS AND A LOCAL PROPERTY OF LIE GROUPS
}

\author{
NAZIH NAHLUS
}

(Communicated by Roe Goodman)

\begin{abstract}
Let $G$ be any analytic group, let $T$ be a maximal toroid of the radical of $G$, and let $S$ be a maximal semisimple analytic subgroup of $G$.

If $L=\mathcal{L}(G)$ is the Lie algebra of $G, \operatorname{rad}[L, L]$ is the radical of $[L, L]$, and $\mathcal{Z}(L)$ is the center of $L$, we show that $G$ has a faithful representation if and only if

(i) $\operatorname{rad}[L, L] \cap \mathcal{Z}(L) \cap \mathcal{L}(T)=(0)$, and

(ii) $S$ has a faithful representation.
\end{abstract}

A theorem of M. Moskowitz [4, Thm. 2], shows that if $L$ is a finite-dimensional (real) Lie algebra, then all analytic groups with Lie algebra $L$ have faithful representations if and only if (i) $\operatorname{rad}[L, L] \cap \mathcal{Z}(L)=(0)$, and (ii) for some maximal semisimple subalgebra $S$ of $L$, the simply connected analytic group with Lie algebra $S$ has a faithful representation. So it would be of interest to find a similar criterion for a single analytic group $G$ to have a faithful representation. Such a criterion is given in Theorem 2 below. As a consequence, we obtain Moskowitz' Theorem in Corollary 3. So our criterion in the solvable case says that $G$ has a faithful representation if and only if $[L, L] \cap \mathcal{Z}(L) \cap \mathcal{L}(T)=(0)$ for some maximal toroid $T$ of $G$ where $L=\mathcal{L}(G)$; whereas the well-known criterion in the solvable case is that $G$ has a faithful representation if and only if $[G, G]$ is closed in $G$ and simply connected $[2$, p. 220]. For the case of semisimple analytic groups, we refer the reader to [2, pp. 199-201].

Our proof uses the notion of nuclei of analytic groups introduced by Hochschild and Mostow. A nucleus $K$ of an analytic group $G$ is a closed normal simply connected solvable analytic subgroup of $G$ such that $G / K$ is reductive. An analytic group $G$ has a faithful representation if and only if $G$ has a nucleus; if $G$ has a nucleus $K$, then $G=K \cdot P$ (semi-direct) for every maximal reductive analytic subgroup $P$ of $G$ [3, Section 2]. Recall that an analytic group is reductive if it has a faithful representation and all its representations are semisimple.

If $G$ is an analytic group, $\mathcal{L}(G)$ is its Lie algebra, $\operatorname{rad} G$ is its radical, and $[G, G]$ is its commutator (derived) subgroup. Similarly, if $L$ is a Lie algebra, $\operatorname{rad} L$ is its radical, and $[L, L]$ is its commutator (derived) subalgebra. All representations of analytic groups are assumed to be analytic and finite dimensional.

Received by the editors October 26, 1995 and, in revised form, March 29, 1996.

1991 Mathematics Subject Classification. Primary 22E15, 22E60.

(C) 1997 American Mathematical Society 
Lemma 1. Let $G$ be any analytic group, and let $A$ and $B$ be analytic subgroups of $G$ such that $A$ is normal in $G$. If $G=A B$ and $A \cap B=(1)$, then $A$ and $B$ are closed in $G$.

Proof. Let $G^{+}=A \times B$ be the cartesian product of the analytic manifolds of $A$ and $B$ underlying the analytic groups $A$ and $B$. Then, in addition to its manifold structure, $G^{+}=A \times B$ is also an abstract group via the conjugation action of $B$ on $A$ in $G$. We now show that these structures turn $G^{+}$into an analytic group. Let $f: A \times B \rightarrow A$ be the mapping given by $f(a, b)=b a b^{-1}$. Then one can easily check that $f$ is analytic on a neighborhood of $\left(1_{A}, 1_{B}\right)$ by using the exponential maps in the analytic groups $A, B$ and $G$. Since $A$ is connected, it follows that $f$ is analytic on $A \times B$ [1, Lemma 3, p. 362].

Hence $G^{+}=A \times B$ is an analytic group with the above group and manifold structures. Now let $p: G^{+}=A \times B \rightarrow A B=G$ be the mapping given by $p(a, b)=a b$. Then $p$ is a surjective continuous homomorphism between locally compact connected topological groups, so $p$ must be an open map [2, Thm. 2.5, p. 7] or [2, Exercise 1, p. 13]. But $p$ is also bijective since $A \cap B=(1)$. Consequently, $p: G^{+} \rightarrow G$ is an isomorphism of analytic groups. Hence $A$ and $B$ are closed in $G$ since they are closed in $G^{+}$.

We shall need the fact that if $G$ has a faithful representation, then its representation radical $N=\operatorname{rad}[G, G]$ is closed in $G$ and simply connected. This is true, for example, because $N$ is contained in every nucleus $K$ of $G$ [3, Section 2] and each $K$ is a closed simply connected solvable analytic subgroup of $G$. For a direct proof, see the proof of Theorem 1 in [4].

Theorem 2. Let $G$ be any analytic group with Lie algebra $L$, let $T$ be a maximal toroid of $\operatorname{rad}(G)$, and let $S$ be a maximal semisimple analytic subgroup of $G$. Then $G$ has a faithful representation if and only if

(i) $\operatorname{rad}[L, L] \cap \mathcal{Z}(L) \cap \mathcal{L}(T)=(0)$, and

(ii) $S$ has a faithful representation.

Proof. Let Ad and ad be the adjoint representations of $G$ and $\mathcal{L}(G)$ respectively on the Lie algebra $L$ of $G$. Since $\operatorname{rad}[L, L]$ acts nilpotently on any representation space of $L[2$, Thm. 3.2, p. 128], ad( $\operatorname{rad}[L, L])$ consists of nilpotent elements. Since $\operatorname{ad}(\mathcal{L}(T))=\mathcal{L}(\operatorname{Ad}(T))$ and $T$ is a toroid, it follows that $\operatorname{ad}(\mathcal{L}(T))$ consists of semisimple elements. Hence ad $(\operatorname{rad}[L, L] \cap \mathcal{L}(T))=(0)$. Thus $(\operatorname{rad}[L, L] \cap \mathcal{L}(T)) \subseteq$ $\mathcal{Z}(L)$. Since $\operatorname{rad}[L, L]=\mathcal{L}(N)$ where $N=\operatorname{rad}[G, G][2$, Thm. 3.1, p. 138], it follows that $\operatorname{rad}[L, L] \cap \mathcal{Z}(L) \cap \mathcal{L}(T)=(0)$ if and only if $\mathcal{L}(N) \cap \mathcal{L}(T)=(0)$.

So first suppose that $G$ has a faithful representation. Then $S$ has a faithful representation. Moreover, as remarked above, $N$ is a closed simply connected solvable analytic subgroup of $G$, so $N$ has no non-trivial compact subgroups [2, Thm. 2.3, p. 138]. Hence $\mathcal{L}(N) \cap \mathcal{L}(T)=(0)$.

Conversely, suppose $\mathcal{L}(N) \cap \mathcal{L}(T)=(0)$. Then there exists a subspace $\underline{K}$ of $\operatorname{rad} \mathcal{L}(G)$ containing $\mathcal{L}(N)$ such that $\operatorname{rad} \mathcal{L}(G)=\underline{K} \oplus \mathcal{L}(T)$. Since $\underline{K}$ contains $\mathcal{L}(N)=\operatorname{rad}[L, L]$ and $\operatorname{rad}[L, L]=[L, \operatorname{rad} L][2, \operatorname{Thm} .3 .2$, p. 128], it follows that $\underline{K}$ is an ideal of $\mathcal{L}(G)$. Hence $\operatorname{rad} \mathcal{L}(G)=\underline{K}+\mathcal{L}(T)$ (semi-direct). Thus if $K$ is the analytic subgroup of $G$ corresponding to $\underline{K}$, then $K$ is normal in $G, \operatorname{rad}(G)=K \cdot T$, and the subgroup $K \cap T$ is discrete in the analytic group $K$. Thus the projection morphism $K \rightarrow K /(K \cap T)$ is a covering. Moreover, $K /(K \cap T)$ is homeomorphic to $\operatorname{rad}(G) / T$ which is known to be a simply connected homogeneous space since $T$ 
is a maximal toroid of $\operatorname{rad}(G)[2$, Exercise 1, p. 187]. Hence the covering morphism $K \rightarrow K /(K \cap T)$ is a homeomorphism of simply connected homogeneous spaces. Thus $K \cap T=(1)$ and $K$ is simply connected. Since $\operatorname{rad}(G)=K \cdot T$, it follows by Lemma 1 that $K$ is closed in $\operatorname{rad}(G)$. Hence $K$ is a nucleus of $\operatorname{rad}(G)$. Consequently, $\operatorname{rad}(G)$ has a faithful representation [3, Section 2]. Since $S$ has also a faithful representation, it follows that $G$ has a faithful representation [2, Thm. 4.2, p. 221]. This proves Theorem 2 .

Corollary 3 (Thm. 2 of [4]). Let L be a finite-dimensional (real) Lie algebra. Let $S$ be a maximal semisimple subalgebra of $L$, and let $S^{*}$ be the simply connected analytic group with Lie algebra $S$. Then all analytic groups with Lie algebra $L$ have faithful representations if and only if

(i) $\operatorname{rad}[L, L] \cap \mathcal{Z}(L)=(0)$, and

(ii) $S^{*}$ has a faithful representation.

Proof. Suppose $\operatorname{rad}[L, L] \cap \mathcal{Z}(L)=(0)$, and $S^{*}$ has a faithful representation. Let $G$ be any analytic group with Lie algebra $L$, and let $S_{g}$ be the (maximal) semisimple analytic subgroup of $G$ corresponding to the Lie algebra $S$. Since $S^{*}$ has a faithful representation, it follows that $S_{g}$ also has a faithful representation [4, Cor. 1a]. Hence $G$ has a faithful representation by Theorem 2 .

For the converse, we may use the proof in [4, top of p. 197] since it refers only to the fact that $N=\operatorname{rad}[G, G]$ is simply connected whenever $G$ has a faithful representation.

\section{REFERENCES}

1. N. Bourbaki, Elements of Mathematics, Lie Groups and Lie Algebras, Chapters 1-3, SpringerVerlag, 1989. MR 89k:17001

2. G. Hochschild, The Structure of Lie Groups, San Francisco: Holden Day, 1965. MR 34:7696

3. G. Hochschild and G. D. Mostow, On the algebra of representative functions of an analytic group, Amer. J. Math., 83 (1961), 111-136. MR 25:5129

4. M. Moskowitz, Faithful representations and a local property of Lie groups, Math. Z., 143 (1975), 193-198. MR 51:10542

Department of Mathematics, American University of Beirut, c/o New York Office, 850 Third Ave., 18Th FloOr, New York, New York 10022

E-mail address: nahlus@layla.aub.edu.lb 